\title{
Type-2 Fuzzy Alpha-cuts
}

Hussam Hamrawi

\author{
College of Computer Sciences \\ University of Bahri \\ Khartoum, Sudan \\ Email: hussamw@bahri.edu.sd
}

\author{
Simon Coupland \\ Robert John \\ Centre for Computational Intelligence Automated Scheduling Optimisation and Planning \\ De Montfort University \\ Leicester, UK \\ Email: simonc@dmu.ac.uk
}

Type-2 fuzzy logic systems make use of type-2 fuzzy sets. To be able to deliver useful type-2 fuzzy logic applications we need to be able to perform meaningful operations on these sets. These operations should also be practically tractable. However, type-2 fuzzy sets suffer the shortcoming of being complex by definition. Indeed, the third dimension, which is the source of extra parameters, is in itself the origin of extra computational cost. The quest for a representation that allow practical systems to be implemented is the motivation for our work. In this paper we define the alpha-cut decomposition theorem for type2 fuzzy sets which is a new representation analogous to the alpha-cut representation of type-1 fuzzy sets and the extension principle. We show that this new decomposition theorem forms a methodology for extending mathematical concepts from crisp sets to type-2 fuzzy sets directly. In the process of developing this theory we also define a generalisation that allows us to extend operations from interval type-2 fuzzy sets or interval valued fuzzy sets to type-2 fuzzy sets. These results will allow for the more applications of type-2 fuzzy sets by expiating the parallelism that the research here affords.

\section{INTRODUCTION}

Zadeh [33]-[35] defined the type-2 fuzzy set (T2FS) along with a plethora of concepts and mathematical functions including the extension principle (EP) and resolution identity more commonly known as the $\alpha$-cut decomposition theorem. The EP extends point-valued operations from the crisp mathematical setting to a corresponding fuzzy mathematical setting, essentially fuzzifying classical mathematical concepts. The $\alpha$-cut decomposition theorem also allows the same extension to be performed in a set-valued manner. The idea is to decompose fuzzy sets into a collection of crisp sets related together via the $\alpha$ levels. This decomposition theorem has been extended to fuzzy sets with interval membership grades known either by interval valued fuzzy sets (IVFSs) or interval T2FSs (IT2FSs) [20]. Type-2 fuzzy sets, (both general and interval), have attracted much attention amongst researchers both in theory and applications (e.g. [3], [5], [8], [13], [14], [24], [25], [28], [29]) mainly for the extra dimension they exhibit, which gives these sets the potential to model extra uncertainty based information. To be able to make use of T2FSs, we should be able to perform meaningful operations on these sets and these operations should also be practically tractable. T2FSs suffer the shortcoming of being complex by definition. Indeed, the third dimension, which is the source of extra parameters, is in itself the origin of extra computational cost. The quest for a representation that allow practical systems to be implemented is a fertile field of research. There are four main representation theorems for T2FSs, in which practical applications and theoretical definition have been investigated. The vertical slice, wavy slice [22], alpha-plane (or zSlices) [17], [27] and geometric [5] representations. Zadeh [33] was the first to define operations for T2FSs, utilising $\alpha$-cuts of each fuzzy membership grade. Recently, Chen and Kawase [4], Tahayori et al. [26], Liu et al. [17], [19], and Wagner and Hagras [27], [28] focused their attention towards decomposing T2FSs into several IVFSs. In particular, Liu [17] defined $\alpha$-planes and Wagner and Hagras [27] defined zSlices as part of their effort to calculate the Centroid of T2FSs. In his work, Liu concluded that the union, intersection and centroid of T2FSs is equal to their respective operations of its constituent $\alpha$-planes. Wagner and Hagras independently concluded the same. Hamrawi and Coupland [9], [10] derived arithmetic operations and defined non-specificity for T2FSs using the same concept and stated a generalised formula in [11], [12]. In this paper we investigate the use of the concept of $\alpha$-cuts and its extension principle for T2FSs. We explain, step by step, the development phases of the theory and definitions. We believe it is a significant step forward in the theory and application of T2FSs. The novel ideas provided in this paper, are themselves built upon existing theories and definitions well accepted in the literature and is an extension to available and definitions. We show how operations on general type-2 fuzzy sets can be broken down into a collection of interval type- 2 or crisp interval operations. The paper is organised as follows: Section 2 provides the notations and necessary back ground for the following work; Section 3 revisits the $\alpha$ plane representation and defines the $\alpha$-plane extension principle; Section 4 discusses the $\alpha$-cut representation of IVFSs; Section 5 defines the $\alpha$-cut representation for T2FSs and the extension principle associated with this representation; Section 6 provides a conclusion.

\section{DeFinitions}

\section{A. Basic Definitions}

In this section we present the notation and definitions used throughout the paper. Let $A$ be a crisp subset of the universe 
$X$, it is a function $A: X \rightarrow\{0,1\}$ that assigns 1 to elements of the domain that belong to $A$ and 0 otherwise. Let $\boldsymbol{C}(\boldsymbol{X})$ be the set of all crisp subsets of $X^{1}$. Let $A$ be an Interval over $X$. It is defined by $A=[\underline{x}, \bar{x}]$ where $\underline{x}, \bar{x} \in X$ and $\underline{x} \leq \bar{x}$. Also let $\boldsymbol{I}(\boldsymbol{X})$ be the set of all interval subsets of $X$. Note that an interval is a special crisp set with $A(x)=1$, $\underline{x} \leq x \leq \bar{x}$ and 0 otherwise. Let a type-1 fuzzy set (T1FS) $A$ be a subset of $X$, and defined to be a function $A: X \rightarrow[0,1]$. It is a generalisation of both crisp sets and intervals. We call a T1FS, a fuzzy set (FS) for short. Let $\boldsymbol{F}(\boldsymbol{X})$ be the set of all fuzzy subsets of $X$, and all FSs defined in this paper be convex. In this paper we are particularly interested in the $\alpha$-cut representation of FSs. The $\alpha$-cut of FS, $A$ on the domain $X$, is a crisp set defined to be $A_{\alpha}=\{x \mid A(x) \geq \alpha\}$, $\alpha \in[0,1], x \in X$. Each $\alpha$-cut is associated with a special FS, $\alpha A_{\alpha} \in \boldsymbol{F}(\boldsymbol{X})$, and called $\alpha$-FS. It is defined such that $\alpha A_{\alpha}(x)=\alpha \wedge A_{\alpha}(x), \forall x$ [16], [23], [32]. Then the $\alpha$-cut representation theorem $(\alpha-\mathrm{RT})$ [16] is defined to be the union of all such $\alpha$-FSs, i.e., $A=\bigcup_{\forall \alpha} \alpha A_{\alpha}$. It is evident that the membership grade of each domain value, $x$, can be calculated by $A(x)=\sup _{\forall \alpha} \alpha A_{\alpha}(x)$. If the FS, $A$, is continuous then its $\alpha$-cut is an interval, $A_{\alpha} \in \boldsymbol{I}(\boldsymbol{X})$, which can be written $A_{\alpha}=\left[\underline{x}_{\alpha}, \bar{x}_{\alpha}\right]$. The strong $\alpha$-cut is another useful crisp set defined to be $A_{\alpha^{+}}=\{x \mid A(x)>\alpha\}$ [16]. In order to define operations for FSs, Zadeh defined the extension principle (EP), in which a function is extended from crisp sets to FSs in a compositional relation between point-values. This EP is sometimes referred to as the sup - min composition. Let, $X=X_{1} \times \ldots \times X_{n}$, be the Cartesian product of universes, and $A_{1}, \ldots, A_{n}$ be FSs in each universe respectively. Also let $Y$ be another universe and $B \in Y$ be a FS such that $B=f\left(A_{1}, \ldots, A_{n}\right)$, where $f: X \rightarrow Y$. Then the EP is defined as follows [33]:

$$
B \Leftrightarrow B(y)=\sup _{\left(x_{1}, \ldots, x_{n}\right) \in f^{-1}(y)} \min \left(A_{1}\left(x_{1}\right), \ldots, A_{n}\left(x_{n}\right)\right)
$$

where $f^{-1}(y)$ is the inverse function of $y=f\left(x_{1}, \ldots, x_{n}\right)$. Zadeh also defined the $\alpha$-cut version of the EP $(\alpha$-EP) to extend operations from crisp sets to FSs directly in a setvalued method. It is defined as follows $[33]^{2}$ :

$$
B=f\left(A_{1}, \ldots, A_{n}\right)=\bigcup_{\forall \alpha} \alpha f\left(A_{1_{\alpha}}, \ldots, A_{n_{\alpha}}\right)
$$

Some researchers have asserted that equations (1) and (2) are equal [1], [23], [33]. An interval valued fuzzy set (IVFS), $\hat{A}$, over $X$ is defined by a function $\hat{A}: X \rightarrow \mathbf{I}([0,1])$, then $\hat{A}(x)=\left[\underline{u}_{x}, \bar{u}_{x}\right]$ and we let, $\hat{F}(\boldsymbol{X})$, be the set of all IVFSs on $X$. The upper membership function (UMF) of an IVFS, $\hat{A}$, is a fuzzy set, $\bar{A}$, where $\bar{A}(x)=\bar{u}_{x}, \forall x$. The lower membership function (LMF) of an IVFS, $\hat{A}$, is a fuzzy set, $\underline{A}$, where $\underline{A}(x)=\underline{u}_{x}, \forall x$. We can see that an IVFS is completely determined by the LMF and UMF, i.e.,

\footnotetext{
${ }^{1}$ This is the powerset in classical set theory. We use a different notation to allow us to easily distinguish between the powerset of crisp value, intervals and fuzzy sets.

${ }^{2}$ Throughout this paper we use $\bigcup$ to denote both crisp and fuzzy union. When used in a fuzzy union we are referring to the maximum t-conorm.
}

$\hat{A}=(\underline{A}, \bar{A})$ which means $\hat{A}(x)=[\underline{A}(x), \bar{A}(x)], \forall x$. Let, $X=X_{1} \times \ldots \times X_{n}$, be the Cartesian product of universes, and $\hat{A}_{1}, \ldots, \hat{A}_{n}$ be IVFSs in each universe respectively. Also let $Y$ be another universe and $\hat{B} \in Y$ be an IVFS such that $\hat{B}=f\left(\hat{A}_{1}, \ldots, \hat{A}_{n}\right)$, where $f: X \rightarrow Y$ is a monotonic mapping. Then to use the Extension Principle with IVFSs, the (IVEP) can be defined as follows:

$$
\hat{B} \equiv(\underline{B}, \bar{B})=\left(f\left(\underline{A}_{1}, \ldots, \underline{A}_{n}\right), f\left(\bar{A}_{1}, \ldots, \bar{A}_{n}\right)\right)
$$

This means, to derive operations for IVFSs we only need to derive operations for their upper and lower membership functions ${ }^{3}$.

\section{B. Type-2 Fuzzy Set Definitions}

In this section we review the main definitions of T2FSs. Let $\tilde{A}$ be a T2FS in the universe $X$. It is a function $\tilde{A}: X \rightarrow \boldsymbol{F}([\mathbf{0 , 1}])$, so the membership grade of each domain value of the T2FS is a FS defined on the unit interval, i.e., $\tilde{A}(x) \in \boldsymbol{F}([\mathbf{0}, \mathbf{1}])$. The vertical slice (VS) [18], [22], i.e., $\tilde{A}_{x} \equiv \tilde{A}(x)$ is a FS with domain values $u_{x} \in[0,1]$ called the primary grades (PGs) and membership grades $\tilde{A}_{x}\left(u_{x}\right) \in[0,1]$ called the secondary grades (SGs). Each PG is associated with one SG and the union of all the primary grades $u_{x}$ of domain value $x$ is called the primary membership (PM), i.e., $J_{x}=\left\{u_{1 x}, u_{2 x}, \ldots, u_{q_{x}}\right\}$ if the domain of membership grades of the T2FS is discrete, and $J_{x}=\left[\underline{u}_{x}, \bar{u}_{x}\right]$ if it is continuous. Normally, it is assumed that the PMs are intervals, and in the discrete case the PM can be calculated by considering the lower and upper bounds, i.e., $J_{x}=\left[\inf _{i=1, \ldots, q} u_{i x}, \sup _{i=1, \ldots, q} u_{i x}\right]$, and hence $J_{x} \in$ $\mathbf{I}([\mathbf{0 , 1}])$. The union of all primary memberships is called the footprint of uncertainty (FOU), $F O U(\tilde{A})=\bigcup_{\forall x}\left(x, J_{x}\right)$. A T2FS can be represented by the union of all its VSs which is called the vertical slice representation, i.e.,

$$
\tilde{A}=\bigcup_{\forall x}\left(x, \tilde{A}_{x}\right)
$$

An interval type-2 fuzzy set (IT2FS) is a T2FS where all the secondary grades are at unity, i.e., $\tilde{A}_{x}\left(u_{x}\right)=1, \forall u_{x} \in$ $J_{x}, \forall x \in X$. It is well known that an IT2FS can be completely determined using its FOU and it is the same as an IVFS [2], [21]. Recently, Liu [17] proposed the $\alpha$ plane representation of T2FSs, and Wagner and Hagras [27] proposed zSlices. Liu defined an $\alpha$-plane, $\tilde{A}_{\tilde{\alpha}}{ }^{4}$, of a T2FS, $\tilde{A}$, to be the union of the PGs of $\tilde{A}$, whose SGs are greater than or equal to level $\tilde{\alpha}$, i.e.,

$$
\tilde{A}_{\tilde{\alpha}}=\left\{\left(x, u_{x}\right) \mid \tilde{A}_{x}\left(u_{x}\right) \geq \tilde{\alpha}, \forall x, \forall u_{x} \in J_{x}\right\}
$$

Then Liu defines an indicator function, $I_{\tilde{A}_{\tilde{\alpha}}}$, acting on $x \in X$ such that,

$$
I_{\tilde{A}_{\tilde{\alpha}}}\left(x, u_{x}\right)= \begin{cases}1, & \left(x, u_{x}\right) \in \tilde{A}_{\tilde{\alpha}} \\ 0, & \left(x, u_{x}\right) \notin \tilde{A}_{\tilde{\alpha}}\end{cases}
$$

\footnotetext{
${ }^{3}$ We have provided a proof for the IVEP, see [12] for more details

${ }^{4} \mathrm{We}$ used $\tilde{\alpha}$ to indicate that it is an $\alpha$-plane in the third dimension.
} 
Then a T2FS associated with each $\alpha$-plane, $\tilde{\alpha} \tilde{A}_{\tilde{\alpha}}$, is defined as follows:

$$
\tilde{\alpha} \tilde{A}_{\tilde{\alpha}}=\left\{\left(\left(x, u_{x}\right), \tilde{\alpha} \cdot I_{\tilde{A}_{\tilde{\alpha}}}\left(x, u_{x}\right)\right) \mid \forall x \in X\right\}
$$

Using this definition the T2FS, $\tilde{A}$, is represented by the union of all its associated T2FSs, i.e.,

$$
\tilde{A}=\bigcup_{\forall \tilde{\alpha}} \tilde{\alpha} \tilde{A}_{\tilde{\alpha}}
$$

Note that we do not use an indicator function to define $\alpha$-cuts for FSs, and hence we will provide a different interpretation to that of Liu in Section III of this paper. We define the $\alpha$-plane RT in an analogous way to that of the $\alpha$-cut RT for FSs. The EP for T2FSs (T2EP) is defined in a similar way to the FS EP [18]. Let, $X=X_{1} \times \ldots \times X_{n}$, be the Cartesian product of universes, and $\tilde{A}_{1}, \ldots, \tilde{A}_{n}$ be T2FSs in each universe respectively. Also let $Y$ be another universe and $\tilde{B} \in Y$ be a T2FS such that $\tilde{B}=f\left(\tilde{A}_{1}, \ldots, \tilde{A}_{n}\right)$, where $f: X \rightarrow Y$ is a monotone mapping. Then applying the EP to T2FSs (T2EP) lead to the following:

$$
\tilde{B} \Leftrightarrow \tilde{B}(y)=\sup _{\left(x_{1}, \ldots, x_{n}\right) \in f^{-1}(y)} \min \left(\tilde{A}_{1}\left(x_{1}\right), \ldots, \tilde{A}_{n}\left(x_{n}\right)\right)
$$

where $y=f\left(x_{1}, \ldots, x_{n}\right)$, and $\tilde{A}_{1}\left(x_{1}\right), \ldots, \tilde{A}_{n}\left(x_{n}\right)$ are the VSs which can be written as $\tilde{A}_{1_{x_{1}}}, \ldots, \tilde{A}_{n_{x_{n}}}$. These definitions are used to formulate the $\alpha$-cut representation theorem for T2FSs.

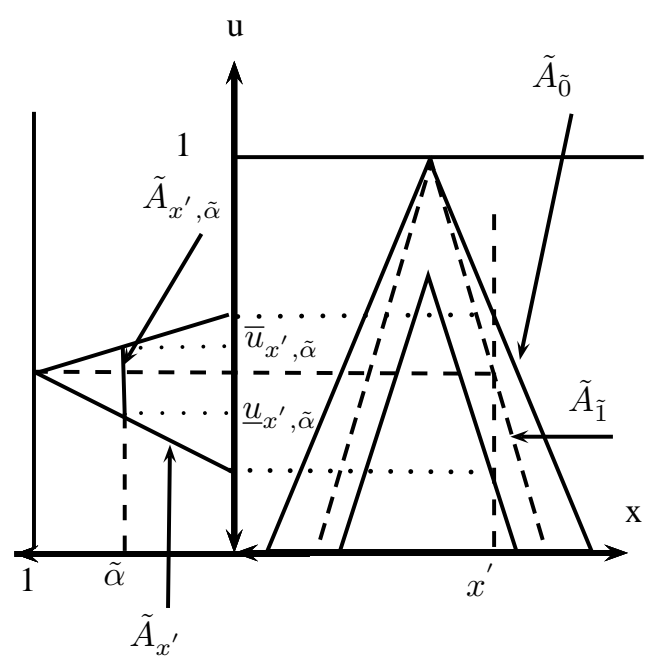

Fig. 1. 2D representation of the T2FS with triangular vertical slices.

\section{T2FS ALPHA-PLANE EXTENSION PRINCIPLE}

In this section we introduce a generalisation that allows us to extend operations from IVFSs to T2FSs directly using $\alpha$-planes. This theory lays the foundation for the $\alpha$-cut decomposition theorem for T2FSs. This method has been stated without a proof by Hamrawi and Coupland [10], with a proof being provided in Hamrawi et al. [11], [12]. Here we start with a discussion on $\alpha$-planes, and the $\alpha$-plane

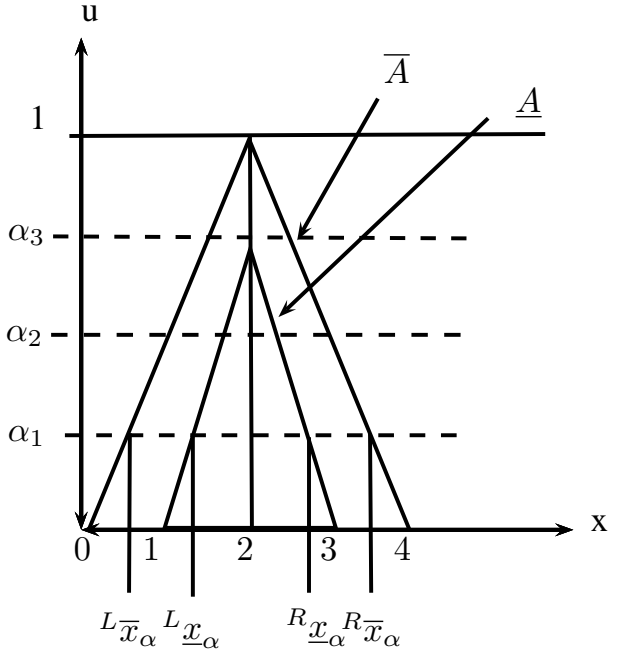

Fig. 2. Continuous IVFS $\hat{A}$ and its $\alpha$-cuts

representation theorem (RT). We investigate some of the properties of these $\alpha$-planes and then define the $\alpha$-plane extension principle ( $\alpha$-PEP).

\section{A. $\alpha$-planes Revisited}

First, the steps Zadeh [33] took in order to define the intersection of two T2FSs are summarised in two stages:

1) Extend the FS definition to fuzzy sets with intervalvalued membership functions.

2) Generalise from intervals to fuzzy sets by the use of the $\alpha$-cut form of the EP ( $\alpha$-EP).

In the sequel, we follow these steps in order to decompose T2FSs into its elementary components, i.e. crisp sets. In general, since each VS is a FS, then it can be decomposed using the $\alpha$-cut decomposition theorem. Let $\tilde{A} \in \tilde{F}(\boldsymbol{X})$ be a T2FS on $X$, where $\tilde{A}_{x}$ is its VS at $x$. The $\alpha$-cuts of each VS are $\tilde{A}_{x, \tilde{\alpha}}=\left\{u_{x} \mid \tilde{A}_{x}\left(u_{x}\right) \geq \tilde{\alpha}\right\}, \forall u_{x} \in J_{x}$. If the domain of the T2FS membership function is assumed to be continuous then $\tilde{A}_{x, \tilde{\alpha}}=\left[\underline{u}_{x, \tilde{\alpha}}, \bar{u}_{x, \tilde{\alpha}}\right]$. Since these VSs are FSs then they can be represented by the $\alpha$-cut decomposition theorem, i.e.,

$$
\tilde{A}_{x}=\bigcup_{\forall \tilde{\alpha}} \tilde{\alpha} \tilde{A}_{x, \tilde{\alpha}}
$$

where $\tilde{\alpha} \tilde{A}_{x, \tilde{\alpha}}$ is the special FS ( $\alpha$-FS) associated with each $\alpha$-cut. It is defined as $\tilde{\alpha} \tilde{A}_{x, \tilde{\alpha}}\left(u_{x}\right)=\tilde{\alpha} \wedge \tilde{A}_{x, \tilde{\alpha}}\left(u_{x}\right)$ and $\tilde{A}_{x, \tilde{\alpha}}\left(u_{x}\right)=1$ if $u_{x} \in \tilde{A}_{x, \tilde{\alpha}}$ and zero otherwise. Then, T2FS $\tilde{A}$ is the union of all its VSs, therefore,

$$
\tilde{A}=\bigcup_{\forall x}\left(x, \bigcup_{\forall \tilde{\alpha}} \tilde{\alpha} \tilde{A}_{x, \tilde{\alpha}}\right)
$$

This is a very important result as a T2FS is represented using a collection of crisp sets (or intervals) defined vertically. Now let us take the union of all the $\alpha$-cuts across all domain values for only one level, i.e., $\bigcup_{\forall x}\left(x, \tilde{A}_{x, \tilde{\alpha}}\right)$. It is the union of all the pairs $\left(x, u_{x}\right)$ such that $\tilde{A}_{x}\left(u_{x}\right) \geq \tilde{\alpha}$. This is exactly the 
same as the $\alpha$-plane definition of equation (5).

$$
\begin{aligned}
\tilde{A}_{\tilde{\alpha}} & =\bigcup_{\forall x}\left(x, \tilde{A}_{x, \tilde{\alpha}}\right) \\
& =\left\{\left(x, u_{x}\right) \mid \tilde{A}_{x}\left(u_{x}\right) \geq \tilde{\alpha}, \forall x, \forall u_{x} \in J_{x}\right\}
\end{aligned}
$$

Here it is clear that

$$
\tilde{A}_{\tilde{\alpha}}\left(x, u_{x}\right)=\tilde{A}_{x, \tilde{\alpha}}\left(u_{x}\right)
$$

We turn our attention to the $\alpha$-FSs of each VS. Let us take the union of all the $\alpha$-FSs across all domain values for only one level, i.e., $\bigcup_{\forall x}\left(x, \tilde{\alpha} \tilde{A}_{x, \tilde{\alpha}}\right)$. It is a T2FS with membership grades $\tilde{\alpha} \tilde{A}_{x, \tilde{\alpha}}$, which are FSs themselves, i.e., $\tilde{\alpha} \tilde{A}_{x, \tilde{\alpha}}=\bigcup_{\forall u_{x}}\left(u_{x}, \tilde{\alpha} \tilde{A}_{x, \tilde{\alpha}}\left(u_{x}\right)\right)$. This is exactly the same as the T2FS associated with each $\alpha$-plane defined in equation (7).

$$
\begin{aligned}
\tilde{\alpha} \tilde{A}_{\tilde{\alpha}} & =\bigcup_{\forall x}\left(x, \tilde{\alpha} \tilde{A}_{x, \tilde{\alpha}}\right) \\
& =\tilde{\alpha} \bigcup_{\forall x}\left(x, \tilde{A}_{x, \tilde{\alpha}}\right) \\
& =\left\{\left(\left(x, u_{x}\right), \tilde{\alpha} \tilde{A}_{\tilde{\alpha}}\left(x, u_{x}\right)\right) \mid \forall x \in X\right\}
\end{aligned}
$$

We call this special T2FS associated with each $\alpha$-plane, $(\alpha$ T2FS), following the same convention we used for FSs. we note that this same definition is called, $\alpha-F O U$ in [19], and $z$ Slice in [27]. We can see that a T2FS is decomposed of these $\alpha$-T2FSs.

Theorem 3.1 ( $\alpha$-Plane RT): A type-2 fuzzy set, $\tilde{A}$, can be represented (decomposed) of the union of all its $\alpha$-T2FSs, i.e.,

$$
\tilde{A}=\bigcup_{\forall \tilde{\alpha}} \tilde{\alpha} \tilde{A}_{\tilde{\alpha}}
$$

Proof. Straight forward from equations (11).(12) and (13). In most cases the $\alpha$-plane, $\tilde{A}_{\tilde{\alpha}}$, is considered to be an IVFS or an IT2FS [17], [19], [27], [28]. This is only the case when the VSs are continuous functions and hence $J_{x} \in \mathbf{I}([0,1])$ is an interval. If the VSs are in discrete domains then as mentioned earlier, the PMs must be bounded through a bounding operation. The following worked example demonstrates how to construct IVFS $\alpha$-planes for discrete T2FSs.

Example 3.1: Let $X=\left\{x_{i} \mid i=1,2, \ldots, 10\right\}$, and very small $(V S), \operatorname{small}(S), \operatorname{medium}(M), \operatorname{large}(L)$, and very large $(V L) \in \boldsymbol{F}([\mathbf{0 , 1}])$ are the FSs that represent the vertical slices, $\tilde{A}_{x}$, defined in Table I. Each vertical slice, $\tilde{A}_{x_{i}}$, consist of PGs, $u_{x_{i}}$, forming its domain and the SGs, $\tilde{A}_{x_{i}}\left(u_{x_{i}}\right)$, forming its membership grade. Let also, $\tilde{A} \in \tilde{F}(\boldsymbol{X})$, be defined as in Table II, with domain values, $x_{i}$, corresponding to vertical slices from Table I. Table III shows how to extract the $\alpha$-cuts $(\tilde{\alpha})$ of the VS $\tilde{A}_{x_{i}}$ of each domain value to form the crisp sets $\tilde{A}_{x_{i}, \tilde{\alpha}}$. Table IV shows how to construct the interval membership grades of the $\alpha$ planes, $\tilde{A}_{\tilde{\alpha}}\left(x_{i}\right)=\left[\min \left(\tilde{A}_{x_{i}, \tilde{\alpha}}\right), \max \left(\tilde{A}_{x_{i}, \tilde{\alpha}}\right)\right]$ in order to formulate the IVFS $\alpha$-planes.
This example demonstrates the case when there are no gaps in the PM, i.e., all VSs are convex. If there is a contrary case, then these sets are approximated to an IVFS using a bounding operation such as taking the minimum and maximum (or infimum and supremum) of the PGs. Note that if these sets are approximated they risk the loss of information. On the other hand, some might argue, what kind of information do such sets hold? In fact most of the reported applications use a structured model of T2FSs that does not involve such sets.

\section{B. T2FS $\alpha$-plane EP}

In this subsection we formulate a theorem that acts as the $\alpha$-based EP for T2FSs. It extends operations from IVFSs to T2FSs, directly. We extend these operations using the $\alpha$ plane RT investigated in the last subsection. Here we state the theorem from Hamrawi et al. [9]-[12].

Theorem $3.2(\alpha-E P)$ : Let, $X=X_{1} \times \ldots \times X_{n}$, be the Cartesian product of universes, and $\tilde{A}_{1}, \ldots, \tilde{A}_{n}$ be T2FSs in each universe respectively. Also let $Y$ be another universe and $\tilde{B} \in Y$ be a T2FS such that $\tilde{B}=f\left(\tilde{A}_{1}, \ldots, \tilde{A}_{n}\right)$, where $f: X \rightarrow Y$ is a monotone mapping. Assume that all the decomposed $\alpha$-planes of all the T2FSs (i.e. $\tilde{A}_{1}, \ldots, \tilde{A}_{n}$ ) are or allowed to be IVFSs. Then $\tilde{B}$ is equal to the union of applying the same function to all the decomposed $\alpha$-planes of $\tilde{A}_{1}, \ldots, \tilde{A}_{n}$, i.e.,

$$
\begin{aligned}
\tilde{B} & =f\left(\tilde{A}_{1}, \ldots, \tilde{A}_{n}\right) \\
& =\bigcup_{\forall \tilde{\alpha}} \tilde{\alpha} f\left(\tilde{A}_{1_{\tilde{\alpha}}}, \ldots, \tilde{A}_{n_{\tilde{\alpha}}}\right)
\end{aligned}
$$

Proof. We start our proof from equation (11)

$$
\tilde{A}_{i}(x)=\bigcup_{\forall \tilde{\alpha}} \tilde{\alpha} \tilde{A}_{i_{x, \tilde{\alpha}}}
$$

where $i=1, \ldots, n$. Then,

$$
\begin{aligned}
\tilde{B}(y) & =f\left(\tilde{A}_{1}, \ldots, \tilde{A}_{n}\right)(y) \\
& =\sup _{\left(x_{1}, \ldots, x_{n}\right)=f^{-1}(y)} \min \left(\tilde{A}_{1}\left(x_{1}\right), \ldots, \tilde{A}_{n}\left(x_{n}\right)\right) \\
& =\sup _{\left(x_{1}, \ldots, x_{n}\right)=f^{-1}(y)} \min \left(\tilde{A}_{1_{x_{1}}}, \ldots, \tilde{A}_{n_{x_{n}}}\right)
\end{aligned}
$$

since $\tilde{A}_{1_{x_{1}}}, \ldots, \tilde{A}_{n_{x_{n}}} \in \boldsymbol{F}(\boldsymbol{X})$ then

$$
\begin{aligned}
\tilde{B}(y) & =\sup _{\left(x_{1}, \ldots, x_{n}\right)=f^{-1}(y)} \min \left(\bigcup_{\forall \tilde{\alpha}} \tilde{\alpha} \tilde{A}_{1_{x_{1}, \tilde{\alpha}}}, \ldots, \bigcup_{\forall \tilde{\alpha}} \tilde{\alpha} \tilde{A}_{n_{x_{n}, \tilde{\alpha}}}\right) \\
& =\sup _{\left(x_{1}, \ldots, x_{n}\right)=f^{-1}(y)} \bigcup_{\forall \tilde{\alpha}} \tilde{\alpha} \min \left(\tilde { A } _ { 1 _ { x _ { 1 } , \tilde { \alpha } } , \ldots , \tilde { A } _ { n _ { x _ { n } , \tilde { \alpha } } } ) } \operatorname { m i n } \left(\tilde{A}_{\left.1_{x_{1}, \tilde{\alpha}}, \ldots, \tilde{A}_{n_{x_{n}, \tilde{\alpha}}}\right)}\right.\right. \\
& =\bigcup_{\forall \tilde{\alpha}} \tilde{\alpha} \sup _{\left(x_{1}, \ldots, x_{n}\right)=f^{-1}(y)} \operatorname{ming}(16)
\end{aligned}
$$


TABLE I

FSS THAT REPRESENT THE VERTICAL SLICES, $\tilde{A}_{x}$, IN EXAMPLE (3.1). THE HORIZENTAL HEADING REPRESENTS THE SGS, $\tilde{A}_{x}\left(u_{x}\right)$, THE VERTICAL HEADING REPRESENTS THE VSS, $\tilde{A}_{x}$, AND THE NUMBERS IN BETWEEN ARE THE PGS, $u_{x}$.

\begin{tabular}{|l|ccccc|}
\hline$\tilde{A}_{x}$ & 0.0 & 0.5 & 1.0 & 0.5 & 0.0 \\
\hline VS & 0.0 & 0.08 & 0.15 & 0.18 & 0.2 \\
S & 0.15 & 0.17 & 0.35 & 0.42 & 0.45 \\
M & 0.4 & 0.43 & 0.5 & 0.6 & 0.65 \\
L & 0.55 & 0.62 & 0.65 & 0.75 & 0.8 \\
VL & 0.7 & 0.78 & 0.85 & 0.9 & 1.0 \\
\hline
\end{tabular}

TABLE II

T2FS, $\tilde{A}$, in EXAMPle (3.1). EACH DOMAin VAlue, $x_{i}$, ALONG With its CORRESPONDing VERTICAL SLICE FROM TABLE (I).

\begin{tabular}{|l|cccccccccc|}
\hline$x_{i}$ & $x_{1}$ & $x_{2}$ & $x_{3}$ & $x_{4}$ & $x_{5}$ & $x_{6}$ & $x_{7}$ & $x_{8}$ & $x_{9}$ & $x_{10}$ \\
\hline$\tilde{A}_{x_{i}}$ & $\mathrm{VS}$ & $\mathrm{VS}$ & $\mathrm{S}$ & $\mathrm{S}$ & $\mathrm{M}$ & $\mathrm{L}$ & $\mathrm{L}$ & $\mathrm{L}$ & $\mathrm{VL}$ & $\mathrm{VL}$ \\
\hline
\end{tabular}

TABLE III

The CRISP SET $\alpha$-CUTS, $\tilde{A}_{x_{i}, \tilde{\alpha}}$, OF THE VERTICAL SLICES,$\tilde{A}_{x_{i}}$, FOR EACH DOMAIN VALUe, $x_{i}$, IN EXAMPLE (3.1)

\begin{tabular}{|l|l|l|l|}
\hline$i$ & $\tilde{\alpha}=0.0$ & $\tilde{\alpha}=0.5$ & $\tilde{\alpha}=1.0$ \\
\hline 1 & $0,0.08,0.15,0.18,0.2$ & $0.08,0.15,0.18$ & 0.15 \\
2 & $0,0.08,0.15,0.18,0.2$ & $0.08,0.15,0.18$ & 0.15 \\
3 & $0.15,0.17,0.35,0.42,0.45$ & $0.17,0.35,0.42$ & 0.35 \\
4 & $0.15,0.17,0.35,0.42,0.45$ & $0.17,0.35,0.42$ & 0.35 \\
5 & $0.4,0.43,0.5,0.6,0.65$ & $0.43,0.5,0.6$ & 0.5 \\
6 & $0.55,0.62,0.65,0.75,0.8$ & $0.62,0.65,0.75$ & 0.65 \\
7 & $0.55,0.62,0.65,0.75,0.8$ & $0.62,0.65,0.75$ & 0.65 \\
8 & $0.55,0.62,0.65,0.75,0.8$ & $0.62,0.65,0.75$ & 0.65 \\
9 & $0.7,0.78,0.85,0.9,1$ & $0.78,0.85,0.9$ & 0.85 \\
10 & $0.7,0.78,0.85,0.9,1$ & $0.78,0.85,0.9$ & 0.85 \\
\hline
\end{tabular}

TABLE IV

THE INTERVAL MEMBERSHIP GRADES OF THE $\alpha$-PLANES, $\tilde{A}_{\tilde{\alpha}}\left(x_{i}\right)$ IN EXAMPLE $(3.1)$

\begin{tabular}{|l|l|l|l|}
\hline$i$ & $\tilde{\alpha}=0.0$ & $\tilde{\alpha}=0.5$ & $\tilde{\alpha}=1.0$ \\
\hline 1 & {$[0,0.2]$} & {$[0.08,0.18]$} & {$[0.15,0.15]$} \\
2 & {$[0,0.2]$} & {$[0.08,0.18]$} & {$[0.15,0.15]$} \\
3 & {$[0.15,0.45]$} & {$[0.17,0.42]$} & {$[0.35,0.35]$} \\
4 & {$[0.15,0.45]$} & {$[0.17,0.42]$} & {$[0.35,0.35]$} \\
5 & {$[0.4,0.65]$} & {$[0.43,0.6]$} & {$[0.5,0.5]$} \\
6 & {$[0.55,0.8]$} & {$[0.62,0.75]$} & {$[0.65,0.65]$} \\
7 & {$[0.55,0.8]$} & {$[0.62,0.75]$} & {$[0.65,0.65]$} \\
8 & {$[0.55,0.8]$} & {$[0.62,0.75]$} & {$[0.65,0.65]$} \\
9 & {$[0.7,1]$} & {$[0.78,0.9]$} & {$[0.85,085]$} \\
10 & {$[0.7,1]$} & {$[0.78,0.9]$} & {$[0.85,0.85]$} \\
\hline
\end{tabular}

now we have $\tilde{A}_{1_{\tilde{\alpha}}}, \ldots, \tilde{A}_{n_{\tilde{\alpha}}} \in \hat{F}(\boldsymbol{X})$, then we substitute each T2FS with its $\alpha$-plane representation

$$
\begin{aligned}
& f\left(\tilde{A}_{1_{\tilde{\alpha}}}, \ldots, \tilde{A}_{n_{\tilde{\alpha}}}\right) \\
& =\sup _{\left(x_{1}, \ldots, x_{n}\right)=f^{-1}(y)} \min \left(\tilde{A}_{1_{\tilde{\alpha}}}\left(x_{1}\right), \ldots, \tilde{A}_{n_{\tilde{\alpha}}\left(x_{n}\right)}\right)
\end{aligned}
$$

then, take the union of all $\tilde{\alpha}$, i.e.,

$$
\begin{aligned}
& f\left(\tilde{A}_{1_{\tilde{\alpha}}}, \ldots, \tilde{A}_{n_{\tilde{\alpha}}}\right) \\
& =\bigcup_{\forall \tilde{\alpha}} \tilde{\alpha} \sup _{\left(x_{1}, \ldots, x_{n}\right)=f^{-1}(y)} \min \left(\tilde{A}_{1_{\tilde{\alpha}}}\left(x_{1}\right), \ldots, \tilde{A}_{n_{\tilde{\alpha}}\left(x_{n}\right)}\right)
\end{aligned}
$$

observe that $\tilde{A}_{i_{\tilde{\alpha}}}\left(x_{i}\right)=\tilde{A}_{i_{x_{i}, \tilde{\alpha}}}, \forall i$, it follows that equations (16) and (17) are equal, and that completes the proof.

The union, the intersection, and the centroid calculation of T2FSs defined by Liu, and Wagner and Hagras can be derived using this theorem. Hamrawi and Coupland [9] defined the non-specificity function in such a way that can be considered a direct implementation of this formula. In all these applications the $\alpha$-planes are considered to be IVFSs. This assumption allows the use of methods already defined for IVFSs (or IT2FSs) with each $\alpha$-plane and thus extended to T2FSs. One of the main advantages of this method is the ability to define operations independently for each $\alpha$-plane. This suggests the use of parallel or distributed techniques to process operations. This $\alpha$-PEP is used to define $\alpha$-cuts for T2FSs. The idea is to make use of the $\alpha$-cut RT for IVFSs and decompose each $\alpha$-plane into $\alpha$-cuts. In the next section we discuss $\alpha$-cuts for IVFSs, in order to be used later to define the $\alpha$-cuts for T2FSs. 


\section{Alpha-Cuts of Interval Valued Fuzzy Sets}

In this section we investigate the $\alpha$-cuts of IVFSs. We already introduced a method for defining $\alpha$-cuts of IVFSs in [11], [12] based on earlier work done by Kaufmann and Gupta [15] on fuzzy arithmetic. It is also related to the aggregation method defined by $\mathrm{Wu}$ and Mendel [30], [31]. Zeng et al. [36], [37] defined a variety of $\alpha$-cut RTs for IVFSs and defined the $\alpha$-EP that makes possible to extend operations from crisp sets to IVFSs directly. Recently, Yager [32] also defined $\alpha$-cuts and the $\alpha$-EP for discrete IVFSs. Figueroa Garcia [6], [7] independently introduced alpha-cuts for type2 interval fuzzy sets, providing an alternative approach to the Karnik-Mendel iterative method for defuzzicafion and for the purposes of formulating and solving linear programming problems. In this section we investigate these methods. We define $\alpha$-cuts for IVFSs by taking the $\alpha$-cut of its LMF and UMF which are themselves FSs, i.e.,

Definition 4.1 (IVFS $\alpha$-cuts): The $\alpha$-cut of an IVFS, $\hat{A}$, is defined as follows:

$$
\hat{A}_{\alpha}=\left(\underline{A}_{\alpha}, \bar{A}_{\alpha}\right)
$$

where $\hat{A}_{\alpha}(x)=\left[\underline{A}_{\alpha}(x), \bar{A}_{\alpha}(x)\right]$.

Note that, the membership of each domain value, $x$, in the set, $\hat{A}_{\alpha}$, is an interval, i.e.,

$$
\hat{A}_{\alpha}(x)= \begin{cases}{[0,0],} & x \notin \underline{A}_{\alpha} \text { and } x \notin \bar{A}_{\alpha} \\ {[0,1],} & x \notin \underline{A}_{\alpha} \text { and } x \in \bar{A}_{\alpha} \\ {[1,1],} & x \in \underline{A}_{\alpha} \text { and } x \in \bar{A}_{\alpha}\end{cases}
$$

These situations are depicted in Figure 3. Notice that we

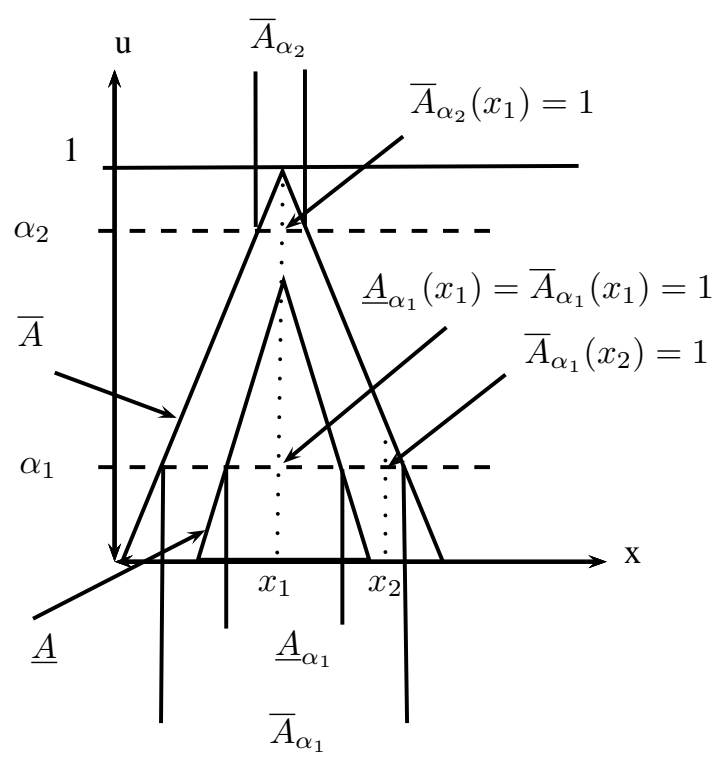

Fig. 3. IVFS $\hat{A}$, its $\operatorname{LMF} \underline{A}$, its $\operatorname{UMF} \bar{A}$ and their $\alpha$-cuts.

did not include a particular impossible situation, that of $\hat{A}_{\alpha}(x)=[1,0]$. This situation is impossible because, by definition, the LMF is always a subset of the UMF, $\underline{A} \subseteq \bar{A}$, i.e., $\underline{A}(x) \leq \bar{A}(x), \forall x$. Which allow us to conclude that $\underline{A}_{\alpha} \subseteq \bar{A}_{\alpha}, \forall \alpha$. The IVFS $\alpha$-cuts are pairs that contain two crisp sets. These sets are treated independently throughout any computation process. This makes it very appealing and holds the semantics of the IVFS definition. The IVFS is actually a FS with an uncertain membership grade which is represented through an interval. The LMF and UMF represents this uncertainty with the interpretation that we do not know exactly the FS, we only know the FS bounds. Again, we follow the same convention of the FS $\alpha$-cuts and define a special IVFS called ( $\alpha$-IVFS) by defining the special FSs $\alpha$-FSs for the LMF and the UMF, i.e.,

Definition $4.2(\alpha-I V F S)$ : A special IVFS ( $\alpha$-IVFS), $\alpha \hat{A}_{\alpha} \in$ $\hat{F}(\boldsymbol{X})$, can be defined as follows:

$$
\begin{aligned}
\alpha \hat{A}_{\alpha} & =\left(\alpha \underline{A}_{\alpha}, \alpha \bar{A}_{\alpha}\right) \\
& =\alpha\left(\underline{A}_{\alpha}, \bar{A}_{\alpha}\right)
\end{aligned}
$$

where $\alpha \hat{A}_{\alpha}(x)=\left[\alpha \wedge \underline{A}_{\alpha}(x), \alpha \wedge \bar{A}_{\alpha}(x)\right]=\alpha \wedge$ $\left[\underline{A}_{\alpha}(x), \bar{A}_{\alpha}(x)\right]$.

Here $\alpha \hat{A}_{\alpha}$ is an IVFS, and each domain value, $x$, is associated with an interval membership grade, $\alpha \hat{A}_{\alpha}(x) \in \mathbf{I}([\mathbf{0 , 1}])$. Also $\alpha \underline{A}_{\alpha}$ and $\alpha \bar{A}_{\alpha}$ are FSs. The $\alpha$-cut RT for IVFSs constitutes the union of all these $\alpha$-IVFSs.

Theorem 4.1 (IVFS $\alpha$-cut RT): An interval valued fuzzy set, $\hat{A}$, can be represented by the following $\alpha$-cut representation theorem:

$$
\hat{A}=\bigcup_{\forall \alpha} \alpha \hat{A}_{\alpha}
$$

Proof. By definition any IVFS is represented using the LMF and UMF, i.e., $\hat{A}=(\underline{A}, \bar{A})$. Since $\underline{A}=\bigcup_{\forall \alpha} \alpha \underline{A}_{\alpha}$ and $\bar{A}=$ $\bigcup_{\forall \alpha} \alpha \bar{A}_{\alpha}$ by the decomposition theorem of FSs, then,

$$
\begin{aligned}
\hat{A} & =\left(\bigcup_{\forall \alpha} \alpha \underline{A}_{\alpha}, \bigcup_{\forall \alpha} \alpha \bar{A}_{\alpha}\right) \\
& =\bigcup_{\forall \alpha}\left(\alpha \underline{A}_{\alpha}, \alpha \bar{A}_{\alpha}\right)
\end{aligned}
$$

Straight forward from definition (4.2) $\alpha \hat{A}_{\alpha}=\left(\alpha \underline{A}_{\alpha}, \alpha \bar{A}_{\alpha}\right)$, and that completes the proof. The following worked example demonstrates how to calculate the $\alpha$-cuts of discrete IVFSs.

Example 4.1: Let $X=\left\{x_{i} \mid i=1,2, \ldots, 10\right\}$, and $\hat{A} \in$ $\hat{F}(\boldsymbol{X})$ is an IVFS defined in Table V. Table VI shows the $\alpha$-cuts of IVFS $\hat{A}$ calculated from its LMF and UMF. Table VII shows how to reconstruct IVFS $\hat{A}$ knowing its $\alpha$-cuts.

Also using equation (20), if $\hat{A}$ is a continuous and convex IVFS i.e. $\underline{A}$ and $\bar{A}$ are continuous and convex as seen in Figure (2). Its $\alpha$-cut is $\hat{A}_{\alpha}=\left(\underline{A}_{\alpha}, \bar{A}_{\alpha}\right)$ where $\underline{A}_{\alpha}=$ $\left[{ }^{L} \underline{x}_{\alpha},{ }^{R} \underline{x}_{\alpha}\right]$ and $\bar{A}_{\alpha}=\left[{ }^{L} \bar{x}_{\alpha}, R \bar{x}_{\alpha}\right]$. Then, $\hat{A}_{\alpha}$, is calculated using the following formula:

$$
\hat{A}_{\alpha}= \begin{cases}\left(\left[{ }^{L} \underline{x}_{\alpha},{ }^{R} \underline{x}_{\alpha}\right],\left[{ }^{L} \bar{x}_{\alpha},{ }^{R} \bar{x}_{\alpha}\right]\right) & , \alpha \leq h(\underline{A}) \\ \left(\emptyset,\left[{ }^{L} \bar{x}_{\alpha},{ }^{R} \bar{x}_{\alpha}\right]\right) & , \alpha>h(\underline{A})\end{cases}
$$


TABLE V

IVFS, $\hat{A}$, IN EXAMPLE (4.1). EACH DOMAIN VALUE, $x_{i}$, ALONG WITH ITS CORRESPONDING INTERVAL MEMBERSHIP GRADE, LMF MEMBERSHIP GRADE AND UMF MEMBERSHIP GRADE.

\begin{tabular}{|l|c|cc|}
\hline$x_{i}$ & $\hat{A}\left(x_{i}\right)$ & $\underline{A}\left(x_{i}\right)$ & $\bar{A}\left(x_{i}\right)$ \\
\hline$x_{1}$ & {$[0,0.6]$} & 0 & 0.6 \\
$x_{2}$ & {$[0,0.8]$} & 0 & 0.8 \\
$x_{3}$ & {$[0,0.9]$} & 0 & 0.9 \\
$x_{4}$ & {$[0.5,1]$} & 0.5 & 1 \\
$x_{5}$ & {$[0.7,1]$} & 0.7 & 1 \\
$x_{6}$ & {$[0.6,1]$} & 0.6 & 1 \\
$x_{7}$ & {$[0.3,0.8]$} & 0.3 & 0.8 \\
$x_{8}$ & {$[0,0.6]$} & 0 & 0.6 \\
$x_{9}$ & {$[0,0.3]$} & 0 & 0.3 \\
$x_{10}$ & {$[0,0.1]$} & 0 & 0.1 \\
\hline
\end{tabular}

TABLE VI

The $\alpha$-CUTS OF IVFS, $\hat{A}$, OF TABLE (V) IN EXAMPLE (4.1).

\begin{tabular}{|l|c|c|}
\hline$\alpha$ & $\underline{A}_{\alpha}$ & $\bar{A}_{\alpha}$ \\
\hline 0.0 & $\left\{x_{1}, x_{2}, x_{3}, x_{4}, x_{5}, x_{6}, x_{7}, x_{8}, x_{9}, x_{10}\right\}$ & $\left\{x_{1}, x_{2}, x_{3}, x_{4}, x_{5}, x_{6}, x_{7}, x_{8}, x_{9}, x_{10}\right\}$ \\
0.1 & $\left\{x_{4}, x_{5}, x_{6}, x_{7}\right\}$ & $\left\{x_{1}, x_{2}, x_{3}, x_{4}, x_{5}, x_{6}, x_{7}, x_{8}, x_{9}, x_{10}\right\}$ \\
0.2 & $\left\{x_{4}, x_{5}, x_{6}, x_{7}\right\}$ & $\left\{x_{1}, x_{2}, x_{3}, x_{4}, x_{5}, x_{6}, x_{7}, x_{8}, x_{9}\right\}$ \\
0.3 & $\left\{x_{4}, x_{5}, x_{6}, x_{7}\right\}$ & $\left\{x_{1}, x_{2}, x_{3}, x_{4}, x_{5}, x_{6}, x_{7}, x_{8}, x_{9}\right\}$ \\
0.4 & $\left\{x_{4}, x_{5}, x_{6}\right\}$ & $\left\{x_{1}, x_{2}, x_{3}, x_{4}, x_{5}, x_{6}, x_{7}, x_{8}\right\}$ \\
0.5 & $\left\{x_{4}, x_{5}, x_{6}\right\}$ & $\left\{x_{1}, x_{2}, x_{3}, x_{4}, x_{5}, x_{6}, x_{7}, x_{8}\right\}$ \\
0.6 & $\left\{x_{5}, x_{6}\right\}$ & $\left\{x_{1}, x_{2}, x_{3}, x_{4}, x_{5}, x_{6}, x_{7}, x_{8}\right\}$ \\
0.7 & $\left\{x_{5}\right\}$ & $\left\{x_{2}, x_{3}, x_{4}, x_{5}, x_{6}, x_{7}\right\}$ \\
0.8 & $\emptyset$ & $\left\{x_{2}, x_{3}, x_{4}, x_{5}, x_{6}, x_{7}\right\}$ \\
0.9 & $\emptyset$ & $\left\{x_{3}, x_{4}, x_{5}, x_{6}\right\}$ \\
1.0 & $\emptyset$ & $\left\{x_{4}, x_{5}, x_{6}\right\}$ \\
\hline
\end{tabular}

TABLE VII

REgenerating IVFS, $\hat{A}$, IN EXAMPLE (4.1) FROM ITS $\alpha$-CUTS IN TABLE (VI)

\begin{tabular}{|l|l|l|l|}
\hline$i$ & $\alpha \underline{A}_{\alpha}\left(x_{i}\right)$ & $\alpha \bar{A}_{\alpha}\left(x_{i}\right)$ & $\hat{A}\left(x_{i}\right)$ \\
\hline 1 & 0 & $0,0.1,0.2,0.3,0.4,0.5,0.6$ & {$[0,0.6]$} \\
2 & 0 & $0,0.1,0.2,0.3,0.4,0.5,0.6,0.7,0.8$ & {$[0,0.8]$} \\
3 & 0 & $0,0.1,0.2,0.3,0.4,0.5,0.6,0.7,0.8,0.9$ & {$[0,0.9]$} \\
4 & $0,0.1,0.2,0.3,0.4,0.5$ & $0,0.1,0.2,0.3,0.4,0.5,0.6,0.7,0.8,0.9,1$ & {$[0.5,1]$} \\
5 & $0,0.1,0.2,0.3,0.4,0.5,0.6,0.7$ & $0,0.1,0.2,0.3,0.4,0.5,0.6,0.7,0.8,0.9,1$ & {$[0.7,1]$} \\
6 & $0,0.1,0.2,0.3,0.4,0.5,0.6$ & $0,0.1,0.2,0.3,0.4,0.5,0.6,0.7,0.8,0.9,1$ & {$[0.6,1]$} \\
7 & $0,0.1,0.2,0.3$ & $0,0.1,0.2,0.3,0.4,0.5,0.6,0.7,0.8$ & {$[0.3,0.8]$} \\
8 & 0 & $0,0.1,0.2,0.3,0.4,0.5,0.6$ & {$[0,0.6]$} \\
9 & 0 & $0,0.1,0.2,0.3$ & {$[0,0.3]$} \\
10 & 0 & $0,0.1$ & {$[0,0.1]$} \\
\hline
\end{tabular}

where $\forall \alpha:{ }^{L} \bar{x}_{\alpha} \leq{ }^{L} \underline{x}_{\alpha} \leq{ }^{R} \underline{x}_{\alpha} \leq{ }^{R} \bar{x}_{\alpha}, h(\underline{A})=\sup _{\forall x} \underline{A}(x)$ is the height of LMF, and $\emptyset$ is an Empty Set. Another way of defining $\alpha$-cuts for IVFSs is the method provided by Kaufmann and Gupta [15]. For example consider the same set provided in equation (22), the $\alpha$-cuts are described in the following way, i.e.,

$$
\hat{A}_{\alpha}^{K G}= \begin{cases}{\left[\left[{ }^{L} \bar{x}_{\alpha},{ }^{L} \underline{x}_{\alpha}\right],\left[{ }^{R} \underline{x}_{\alpha},{ }^{R} \bar{x}_{\alpha}\right]\right]} & , \alpha<h(\underline{A}) \\ {\left[{ }^{L} \bar{x}_{\alpha},{ }^{R} \bar{x}_{\alpha}\right]} & , \alpha \geq h(\underline{A})\end{cases}
$$

There are two drawbacks to this method. Firstly, it does not reduce to the $\alpha$-cut of FSs directly, instead some manipulation and rearrangement must be done and secondly, it does not hold the semantics of $\alpha$-cuts through out the representation. In equation (23), what does $x \in\left[{ }^{L} \bar{x}_{\alpha},{ }^{L} \underline{x}_{\alpha}\right]$ represent? It has a rather complicated relationship to LMF and UMF. It is the values $x$ of the domain that belongs to $\bar{A}_{\alpha}$ and does not belong to non boundary elements of $\underline{A}_{\alpha}$, i.e.,

$$
\begin{aligned}
\hat{A}_{\alpha} & =\left\{x \mid x \in \bar{A}_{\alpha} \text { and } x \notin\left\{\underline{A}_{\alpha}-\left\{\inf _{\forall x} \underline{A}_{\alpha}, \sup _{\forall x} \underline{A}_{\alpha}\right\}\right\}\right\} \\
& =\left\{x \in\left[{ }^{L} \underline{x}_{\alpha},{ }^{R} \underline{x}_{\alpha}\right] \text { and } x \notin\left({ }^{L} \bar{x}_{\alpha},{ }^{R} \bar{x}_{\alpha}\right)\right\} \\
& =\bar{A}_{\alpha} \cap \underline{A}_{\alpha^{+}}^{\prime}
\end{aligned}
$$

where the minus sign - represents the set difference, $\left({ }^{L} \bar{x}_{\alpha},{ }^{R} \bar{x}_{\alpha}\right)$ is an open interval, and $\underline{A}_{\alpha^{+}}^{\prime}$ is the complement of the strong $\alpha$-cut $\left(\alpha^{+}\right)$of the LMF $\underline{A}$. Zeng et al. [36], [37] defined a variety of $\alpha$-cuts. We are interested in one 
particular case, i.e.,

$$
\hat{A}_{\alpha}=\{x \mid \underline{A}(x) \geq \alpha, \bar{A}(x) \geq \alpha\}
$$

Equation 25 is a generalisation of the $\alpha$-cuts for FSs. There is no distinction between the domain values that belong to the $\alpha$-cuts of the LMF and the UMF. Hence, the $\alpha$-cut is a crisp set rather than a pair. Yager [32] also defined a closely related definition for the discrete cases, which can easily be generalised for continuous cases. Although there are different ways to define $\alpha$-cuts for IVFSs, the representation theorem is the same. The ability to extend operations using the $\alpha$-cut RT is what makes it useful.

Theorem 4.2 (IVFS $\alpha$-EP): Let, $X=X_{1} \times \ldots \times X_{n}$, be the Cartesian product of universes, and $\hat{A}_{1}, \ldots, \hat{A}_{n}$ be IVFSs in each universe respectively. Also let $Y$ be another universe and $\hat{B} \in Y$ be an IVFS such that $\hat{B}=f\left(\hat{A}_{1}, \ldots, \hat{A}_{n}\right)$, where $f: X \rightarrow Y$ is a monotonic mapping. Then, $\hat{B}$, is equal to the union of applying the same function to all the decomposed $\alpha$-cuts of the IVFSs [12], i.e.,

$$
\begin{aligned}
\hat{B} & =f\left(\hat{A}_{1}, \ldots, \hat{A}_{n}\right) \\
& =\bigcup_{\forall \alpha} \alpha\left(f\left(\underline{A}_{1_{\alpha}}, \ldots, \underline{A}_{n_{\alpha}}\right), f\left(\bar{A}_{1_{\alpha}}, \ldots, \bar{A}_{n_{\alpha}}\right)\right)
\end{aligned}
$$

Proof. Since $\underline{A}_{1}, \ldots, \underline{A}_{n}, \bar{A}_{1}, \ldots, \bar{A}_{n} \in \boldsymbol{F}(\boldsymbol{X})$, then from equation (2)

$$
\begin{aligned}
f\left(\underline{A}_{1}, \ldots, \underline{A}_{n}\right) & =\bigcup_{\forall \alpha} \alpha f\left(\underline{A}_{1_{\alpha}}, \ldots, \underline{A}_{n_{\alpha}}\right) \\
f\left(\bar{A}_{1}, \ldots, \bar{A}_{n}\right) & =\bigcup_{\forall \alpha} \alpha f\left(\bar{A}_{1_{\alpha}}, \ldots, \bar{A}_{n_{\alpha}}\right)
\end{aligned}
$$

Therefore, we have

$$
\begin{aligned}
f & \left(\hat{A}_{1}, \ldots, \hat{A}_{n}\right)=\left(f\left(\underline{A}_{1}, \ldots, \underline{A}_{n}\right), f\left(\bar{A}_{1}, \ldots, \bar{A}_{n}\right)\right) \\
& =\left(\bigcup_{\forall \alpha} \alpha f\left(\underline{A}_{1_{\alpha}}, \ldots, \underline{A}_{n_{\alpha}}\right), \bigcup_{\forall \alpha} \alpha f\left(\bar{A}_{1_{\alpha}}, \ldots, \bar{A}_{n_{\alpha}}\right)\right) \\
& =\bigcup_{\forall \alpha} \alpha\left(f\left(\underline{A}_{1_{\alpha}}, \ldots, \underline{A}_{n_{\alpha}}\right), f\left(\bar{A}_{1_{\alpha}}, \ldots, \bar{A}_{n_{\alpha}}\right)\right)
\end{aligned}
$$

which completes the proof. $\mathbf{a}$ The following example shows how to perform the union and intersection of IVFSs using $\alpha$-cuts.

Example 4.2: Let $\hat{4}$ and $\hat{8}$ be two IVFS defined in Table VIII and Table IX, respectively. The $\alpha$-cuts of both their LMF and UMF is shown in Table X. The union of the $\alpha$ cuts are shown in Table XI. This will eventually lead to an IVFS $\hat{4} \cup \hat{8}$. The method used to generate the membership grades of $\hat{4} \cup \hat{8}$ from its $\alpha$-cuts is shown in Table XII. The intersection of the $\alpha$-cuts are shown in Table XIII. This will eventually lead to an IVFS $\hat{4} \cap \hat{8}$. The method used to generate the membership grades of $\hat{4} \cap \hat{8}$ from its $\alpha$-cuts is shown in Table XIV.
To summarise the overall picture, we view the process of deriving operations for IVFSs to involve the definition of these operations for two distinct FSs, i.e., the UMF and LMF. The same operations can be defined for crisp sets (or intervals) and then extend them to FSs using the $\alpha$-EP. The obvious conclusion is to define these operations for IVFSs by taking both FSs and using the $\alpha$-EP. To derive operations for IVFSs in such a simple and elegant process is in itself, we believe, a significant result.

\section{Alpha-Cuts of TyPE-2 FuZZY Sets}

\section{A. $\alpha$-cut Representation Theorem}

In the previous section we discussed $\alpha$-cuts for IVFSs. These $\alpha$-cuts can be defined in different ways. What is important, is that these are crisp sets and the IVFS $\alpha$ EP extends operations directly from crisp sets to IVFSs. This fact is crucial since in Section III we showed that $\alpha$ planes are IVFSs, and developed the $\alpha$-PEP to allow us to extend operations from IVFSs to T2FSs. Combining these two theorems lead us to define $\alpha$-cuts for T2FSs, directly. First, we define the UMF and LMF of $\alpha$-planes.

Definition 5.1: Let, $\tilde{A} \in \tilde{F}(\boldsymbol{X})$, be a T2FS and, $\tilde{A}_{\tilde{\alpha}} \in$ $\hat{F}(\boldsymbol{X})$, be a IVFS representing its $\alpha$-plane at level $\tilde{\alpha}$, such that $\tilde{A}_{\tilde{\alpha}}=\left[\underline{u}_{x, \tilde{\alpha}}, \bar{u}_{x, \tilde{\alpha}}\right]$. Let, $\underline{A}_{\tilde{\alpha}} \in \boldsymbol{F}(\boldsymbol{X})$, be the LMF of $\tilde{A}_{\tilde{\alpha}}$ and , $\bar{A}_{\tilde{\alpha}} \in \boldsymbol{F}(\boldsymbol{X})$, be the UMF of $\tilde{A}_{\tilde{\alpha}}$. Then each $\alpha$-plane is completely determined by its LMF and UMF, i.e.,

$$
\tilde{A}_{\tilde{\alpha}}=\left(\underline{A}_{\tilde{\alpha}}, \bar{A}_{\tilde{\alpha}}\right)
$$

where $\tilde{A}_{\tilde{\alpha}}(x)=\left[\underline{A}_{\tilde{\alpha}}(x), \bar{A}_{\tilde{\alpha}}(x)\right], \underline{A}_{\tilde{\alpha}}(x)=\underline{u}_{x, \tilde{\alpha}}$ and $\bar{A}_{\tilde{\alpha}}(x)=\bar{u}_{x, \tilde{\alpha}}$.

It is clear that both the LMF and UMF are FSs. Now, let us take the $\alpha$-cuts of each $\alpha$-plane.

Definition 5.2 (T2 $\alpha$-cuts): Let, $\tilde{A} \in \tilde{F}(\boldsymbol{X})$, be a T2FS and, $\tilde{A}_{\tilde{\alpha}}=\left(\underline{A}_{\tilde{\alpha}}, \bar{A}_{\tilde{\alpha}}\right)$, be its $\alpha$-plane at level $\tilde{\alpha}$ represented by its LMF and UMF. Then, $\tilde{A}_{\tilde{\alpha}, \alpha}$, is the $\alpha$-cut of that $\alpha$-plane at level $\alpha$, i.e.,

$$
\tilde{A}_{\tilde{\alpha}, \alpha}=\left(\underline{A}_{\tilde{\alpha}, \alpha}, \bar{A}_{\tilde{\alpha}, \alpha}\right)
$$

where $\underline{A}_{\tilde{\alpha}, \alpha}$ and $\bar{A}_{\tilde{\alpha}, \alpha}$ are the $\alpha$-cuts of the LMF and UMF of $\alpha$-plane, $\tilde{A}_{\tilde{\alpha}}$, respectively.

The LMF and UMF $\alpha$-cuts are crisp sets since the LMF and UMF are FSs. Hence, $\underline{A}_{\tilde{\alpha}, \alpha}(x) \in\{0,1\}$, and $\bar{A}_{\tilde{\alpha}, \alpha}(x) \in$ $\{0,1\}$. Following definition (4.2) we define $\alpha$-IVFS of each $\alpha$-cut, i.e.,

Definition 5.3: For each $\alpha$-cut, $\tilde{A}_{\tilde{\alpha}, \alpha}$, of the T2FS, $\tilde{A}$, a special IVFS ( $\alpha$-IVFS), $\alpha \tilde{A}_{\tilde{\alpha}, \alpha} \in \hat{F}(\boldsymbol{X})$, can be defined as follows:

$$
\begin{aligned}
\alpha \tilde{A}_{\tilde{\alpha}, \alpha} & =\alpha\left(\underline{A}_{\tilde{\alpha}, \alpha}, \bar{A}_{\tilde{\alpha}, \alpha}\right) \\
& =\left(\alpha \underline{A}_{\tilde{\alpha}, \alpha}, \alpha \bar{A}_{\tilde{\alpha}, \alpha}\right)
\end{aligned}
$$


TABLE VIII

IVFS, $\hat{4}$, IN EXAMPLE 4.2

\begin{tabular}{|l|ccccc|}
\hline$x$ & 2 & 3 & 4 & 5 & 6 \\
\hline$\hat{4}(x)$ & {$[0,0.2]$} & {$[0.4,0.6]$} & {$[0.8,1]$} & {$[0.5,0.6]$} & {$[0,0.4]$} \\
\hline
\end{tabular}

TABLE IX

IVFS, $\hat{8}$, IN EXAMPLE 4.2 .

\begin{tabular}{|l|ccccccc|}
\hline$x$ & 5 & 6 & 7 & 8 & 9 & 10 & 11 \\
\hline$\hat{8}(x)$ & {$[0,0.1]$} & {$[0.2,0.5]$} & {$[0.6,0.8]$} & {$[1,1]$} & {$[0.5,0.8]$} & {$[0.2,0.4]$} & {$[0,0.1]$} \\
\hline
\end{tabular}

TABLE $X$

THE $\alpha$-CUTS OF IVFS, $\hat{4}$ AND $\hat{8}$, IN EXAMPLE 4.2.

\begin{tabular}{|l|c|c|c|c|}
\hline$\alpha$ & $\underline{4}_{\alpha}$ & $\underline{8}_{\alpha}$ & $\overline{4}_{\alpha}$ & $\overline{8}_{\alpha}$ \\
\hline 0.0 & $\{2,3,4,5,6\}$ & $\{5,6,7,8,9,10,11\}$ & $\{2,3,4,5,6\}$ & $\{5,6,7,8,9,10,11\}$ \\
0.1 & $\{3,4,5\}$ & $\{6,7,8,9,10\}$ & $\{2,3,4,5,6\}$ & $\{5,6,7,8,9,10,11\}$ \\
0.2 & $\{3,4,5\}$ & $\{6,7,8,9,10\}$ & $\{2,3,4,5,6\}$ & $\{6,7,8,9,10\}$ \\
0.3 & $\{3,4,5\}$ & $\{7,8,9\}$ & $\{3,4,5,6\}$ & $\{6,7,8,9,10\}$ \\
0.4 & $\{3,4,5\}$ & $\{7,8,9\}$ & $\{3,4,5,6\}$ & $\{6,7,8,9,10\}$ \\
0.5 & $\{4,5\}$ & $\{7,8,9\}$ & $\{3,4,5\}$ & $\{6,7,8,9\}$ \\
0.6 & $\{4\}$ & $\{7,8\}$ & $\{3,4,5\}$ & $\{7,8,9\}$ \\
0.7 & $\{4\}$ & $\{8\}$ & $\{4\}$ & $\{7,8,9\}$ \\
0.8 & $\{4\}$ & $\{8\}$ & $\{4\}$ & $\{7,8,9\}$ \\
0.9 & $\emptyset$ & $\{8\}$ & $\{4\}$ & $\{8\}$ \\
1.0 & $\emptyset$ & $\{8\}$ & $\{4\}$ & $\{8\}$ \\
\hline
\end{tabular}

TABLE XI

THE $\alpha$-CUTS OF IVFS, $\hat{4} \cup \hat{8}$, IN EXAMPLE 4.2.

\begin{tabular}{|l|c|c|}
\hline$\alpha$ & $\underline{4}_{\alpha} \cup \underline{8}_{\alpha}$ & $\overline{4}_{\alpha} \cup \overline{8}_{\alpha}$ \\
\hline 0.0 & $\{2,3,4,5,6,7,8,9,10,11\}$ & $\{2,3,4,5,6,7,8,9,10,11\}$ \\
0.1 & $\{3,4,5,6,7,8,9,10\}$ & $\{2,3,4,5,6,7,8,9,10,11\}$ \\
0.2 & $\{3,4,5,6,7,8,9,10\}$ & $\{2,3,4,5,6,7,8,9,10\}$ \\
0.3 & $\{3,4,5,7,8,9\}$ & $\{3,4,5,6,7,8,9,10\}$ \\
0.4 & $\{3,4,5,7,8,9\}$ & $\{3,4,5,6,7,8,9,10\}$ \\
0.5 & $\{4,5,7,8,9\}$ & $\{3,4,5,6,7,8,9\}$ \\
0.6 & $\{4,7,8\}$ & $\{3,4,5,7,8,9\}$ \\
0.7 & $\{4,8\}$ & $\{4,7,8,9\}$ \\
0.8 & $\{4,8\}$ & $\{4,7,8,9\}$ \\
0.9 & $\{8\}$ & $\{4,8\}$ \\
1.0 & $\{8\}$ & $\{4,8\}$ \\
\hline
\end{tabular}

where $\alpha \tilde{A}_{\tilde{\alpha}, \alpha}(x)=\alpha \wedge\left[\underline{A}_{\tilde{\alpha}, \alpha}(x), \bar{A}_{\tilde{\alpha}, \alpha}(x)\right]$.

It is noticeable that $\alpha \underline{A}_{\tilde{\alpha}, \alpha}$ and $\alpha \bar{A}_{\tilde{\alpha}, \alpha}$ are special FSs $(\alpha$ FS). The union of all $\alpha$-IVFSs constitute an $\alpha$-plane.

$$
\begin{aligned}
\tilde{A}_{\tilde{\alpha}} & =\bigcup_{\forall \alpha} \alpha \tilde{A}_{\tilde{\alpha}, \alpha} \\
& =\bigcup_{\forall \alpha} \alpha\left(\underline{A}_{\tilde{\alpha}, \alpha}, \bar{A}_{\tilde{\alpha}, \alpha}\right)
\end{aligned}
$$

Earlier in Equation 13 we defined a special T2FS ( $\alpha$-T2FS) associated with each $\alpha$-plane, $\tilde{\alpha} \tilde{A}_{\tilde{\alpha}}$. We make use of this definition again.

$$
\begin{aligned}
\tilde{\alpha} \tilde{A}_{\tilde{\alpha}} & =\tilde{\alpha} \bigcup_{\forall \alpha} \alpha \tilde{A}_{\tilde{\alpha}, \alpha} \\
& =\tilde{\alpha} \bigcup_{\forall \alpha} \alpha\left(\underline{A}_{\tilde{\alpha}, \alpha}, \bar{A}_{\tilde{\alpha}, \alpha}\right)
\end{aligned}
$$

where $\left(\tilde{\alpha} \bigcup_{\forall \alpha} \alpha \tilde{A}_{\tilde{\alpha}, \alpha}(x)\right)\left(u_{x, \tilde{\alpha}}\right)=\quad \tilde{\alpha} \wedge$ $\left(\bigcup_{\forall \alpha} \alpha \tilde{A}_{\tilde{\alpha}, \alpha}(x)\right)\left(u_{x, \tilde{\alpha}}\right)$ and $\bigcup_{\forall \alpha} \alpha \tilde{A}_{\tilde{\alpha}, \alpha}(x)\left(u_{x, \tilde{\alpha}}\right)=1$ if $u_{x, \tilde{\alpha}} \in \bigcup_{\forall \alpha} \alpha \tilde{A}_{\tilde{\alpha}, \alpha}(x)$ and zero otherwise. It is already known from the $\alpha$-plane representation theorem that a T2FS can be represented by the union of all such $\alpha$-T2FSs.

Theorem 5.1 (T2FS $\alpha$-cut RT): A T2FS, $\tilde{A}$, can be represented by the union of all its $\alpha$-T2FSs, i.e.,

$$
\tilde{A}=\bigcup_{\forall \tilde{\alpha}} \tilde{\alpha} \bigcup_{\forall \alpha} \alpha \tilde{A}_{\tilde{\alpha}, \alpha}
$$

Proof. Straight forward substitute equation (31) in equation (14) of theorem (4.1). - The $\alpha$-cut representation allow T2FSs to be decomposed into its smallest interpretable components, i.e., crisp sets while maintaining the relationship between domain values by their degree of membership. T2FSs can be looked upon as weighted crisp sets with the PGs and SGs as weighting factors. The VS, $\alpha$-plane and $\alpha$ - 
TABLE XII

IVFS, $\hat{4} \cup \hat{8}$, IN EXAMPLE 4.2 FROM ITS $\alpha$-CUTS IN TABLE XI.

\begin{tabular}{|l|l|l|l|}
\hline$x$ & $\alpha(\underline{4} \cup \underline{8})_{\alpha}(x)$ & $\alpha(\overline{4} \cup \overline{8})_{\alpha}(x)$ & $(\hat{4} \cup \hat{8})(x)$ \\
\hline 2 & 0 & $0,0.1,0.2$ & {$[0,0.2]$} \\
3 & $0,0.1,0.2,0.3,0.4$ & $0,0.1,0.2,0.3,0.4,0.5,0.6$ & {$[0.4,0.6]$} \\
4 & $0,0.1,0.2,0.3,0.4,0.5,0.6,0.7,0.8$ & $0,0.1,0.2,0.3,0.4,0.5,0.6,0.7,0.8,0.9,1$ & {$[0.8,1]$} \\
5 & $0,0.1,0.2,0.3,0.4,0.5$ & $0,0.1,0.2,0.3,0.4,0.5,0.6$ & {$[0.5,0.6]$} \\
6 & $0,0.1,0.2$ & $0,0.1,0.2,0.3,0.4,0.5$ & {$[0.2,0.5]$} \\
7 & $0,0.1,0.2,0.3,0.4,0.5,0.6$ & $0,0.1,0.2,0.3,0.4,0.5,0.6,0.7,0.8$ & {$[0.6,0.8]$} \\
8 & $0,0.1,0.2,0.3,0.4,0.5,0.6,0.7,0.8,0.9,1$ & $0,0.1,0.2,0.3,0.4,0.5,0.6,0.7,0.8,0.9,1$ & {$[1,1]$} \\
9 & $0,0.1,0.2,0.3,0.4,0.5$ & $0,0.1,0.2,0.3,0.4,0.5,0.6,0.7,0.8$ & {$[0.5,0.8]$} \\
10 & $0,0.1,0.2$ & $0,0.1,0.2,0.3,0.4$ & {$[0.2,0.4]$} \\
11 & 0 & $0,0.1$ & {$[0,0.1]$} \\
\hline
\end{tabular}

TABLE XIII

THE $\alpha$-CUTS OF IVFS, $\hat{4} \cap \hat{8}$, IN EXAMPLE 4.2.

\begin{tabular}{|l|c|c|}
\hline$\alpha$ & $\underline{4}_{\alpha} \cap \underline{8}_{\alpha}$ & $\overline{4}_{\alpha} \cap \overline{8}_{\alpha}$ \\
\hline 0.0 & $\{5,6\}$ & $\{5,6\}$ \\
0.1 & $\emptyset$ & $\{5,6\}$ \\
0.2 & $\emptyset$ & $\{6\}$ \\
0.3 & $\emptyset$ & $\{6\}$ \\
0.4 & $\emptyset$ & $\{6\}$ \\
0.5 & $\emptyset$ & $\emptyset$ \\
0.6 & $\emptyset$ & $\emptyset$ \\
0.7 & $\emptyset$ & $\emptyset$ \\
0.8 & $\emptyset$ & $\emptyset$ \\
0.9 & $\emptyset$ & $\emptyset$ \\
1.0 & $\emptyset$ & $\emptyset$ \\
\hline
\end{tabular}

TABLE XIV

IVFS, $\hat{4} \cap \hat{8}$, IN EXAMPLE 4.2 FROM ITS $\alpha$-CUTS IN TABLE XIII

\begin{tabular}{|l|l|l|l|}
\hline$x$ & $\alpha(\underline{4} \cap \underline{8})_{\alpha}(x)$ & $\alpha(\overline{4} \cap \overline{8})_{\alpha}(x)$ & $(\hat{4} \cap \hat{8})(x)$ \\
\hline 5 & 0 & $0,0.1$ & {$[0,0.1]$} \\
6 & 0 & $0,0.1,0.2,0.3,0.4$ & {$[0,0.4]$} \\
\hline
\end{tabular}

cut representations are by definition related. The relationship between these representations is depicted in Figure 4. The

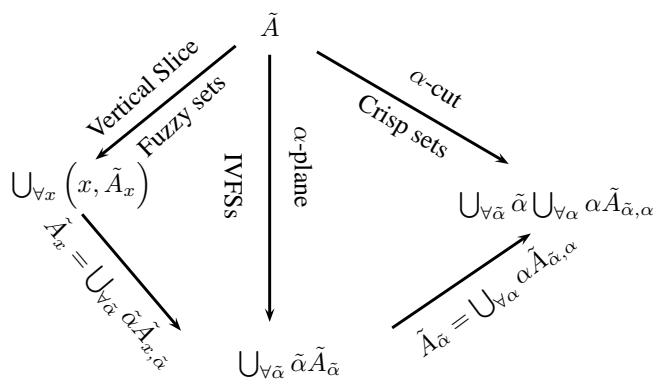

Fig. 4. The vertical slice, $\alpha$-plane and $\alpha$-cut representations of T2FSs and their relationship.

relation between domain values in the classical set theoretic way is behind the idea of $\alpha$-cuts for FSs. This relation is maintained across IVFSs and T2FSs as they are extension of classical FSs. What makes such decomposition interesting is the ability to perform operations in the classical set theoretic sense. This is made possible by extending the $\alpha$-EP of FSs to IVFSs, and by the $\alpha$-PEP of $\alpha$-planes.

Theorem 5.2 (T2FS $\alpha$-cut EP): Let, $X=X_{1} \times \ldots \times X_{n}$, be the Cartesian product of universes, and $\tilde{A}_{1}, \ldots, \tilde{A}_{n}$ be T2FSs in each universe respectively. Also let $Y$ be another universe and $\tilde{B} \in Y$ be a T2FS such that $\tilde{B}=f\left(\tilde{A}_{1}, \ldots, \tilde{A}_{n}\right)$, where $f: X \rightarrow Y$ is a monotone mapping. Then $\tilde{B}$ is equal to the union of applying the same function to all its decomposed $\alpha$-cuts, i.e.,

$$
\begin{aligned}
\tilde{B} & =f\left(\tilde{A}_{1}, \ldots, \tilde{A}_{n}\right) \\
& =\bigcup_{\forall \tilde{\alpha}} \tilde{\alpha} \bigcup_{\forall \alpha} \alpha f\left(\tilde{A}_{1_{\tilde{\alpha}, \alpha}}, \ldots, \tilde{A}_{n_{\tilde{\alpha}, \alpha}}\right)
\end{aligned}
$$

Proof. From theorem (3.2) operations are extended to T2FSs by the $\alpha$-PEP from operations on its $\alpha$-planes which are IVFSs. For each $\alpha$-plane theorem (4.2) allows the operations to be extended from crisp sets. Hence, straight forward substitute equation (26) in equation (15) and that completes the proof. This theorem first appeared in [10]. The following example demonstrates how to use Theorem 5.2 for defining operations for T2FSs by calculating the join and meet of a T2FS using the $\alpha$-cut extension principle.

Example 5.1: Consider the T2FSs, $\tilde{3}$, in Table XV and, $\tilde{6}$, in Table XVI. To perform the join, a decomposition of 
each T2FS into its $\alpha$-planes and each $\alpha$-plane to its $\alpha$-cuts must be performed. Then, for example the union of $\alpha$-planes $\tilde{3}_{0.2} \cup \tilde{6}_{0.2}$, is computed. The interval membership grades of each $\alpha$-plane are constructed using the bounds of the PMs $J_{x, \tilde{\alpha}}$, i.e. Table XVII and Table XVIII. The steps to perform the union is shown in Table XIX, Table XX and Table XXI. These are the same steps used to perform the union of IVFSs. To perform the union of the T2FSs the same task is repeated for all the $\alpha$-planes.

In this section we defined $\alpha$-cuts for T2FSs and its associated T2FS $\alpha$-EP which allows us to extend operations to FSs and its extensions.

\section{CONCLUSION}

In this paper we defined the $\alpha$-cut decomposition theorem for T2FSs, through the use of the basic ideas of $\alpha$-cuts in FSs and the EP. We also showed that this novel decomposition theorem can extend mathematical concepts from crisp sets to T2FSs, directly. In this paper also we investigated a generalisation that allow us to extend operations from IVFSs to T2FSs, through the $\alpha$-plane RT. In order to clarify these concepts we used several worked examples. It is the authors belief that the novel theories provided in this paper will stimulate more investigation and applications of T2FSs. Future work includes taking advantage of the independent nature of these $\alpha$-cuts to perform operations on parallel processors, such as graphical processing units (GPUs).

\section{REFERENCES}

[1] B. Araabi, N. Kehtarnavaz, and C. Lucas. Restrictions imposed by the fuzzy extension of relations and functions. Journal of Intelligent and Fuzzy Systems, 11(1):9-22, 2001.

[2] H. Bustince, E. Barrenechea, M. Pagola, and J. Fernandez. Intervalvalued fuzzy sets constructed from matrices: Application to edge detection. Fuzzy Sets and Systems, 160(13):1819-1840, 2009.

[3] O. Castillo and P. Melin. Type-2 Fuzzy Logic: Theory and Applications, volume 223. Springer-Verlag, Heidelberg, Germany, 1st edition, January 2008. Book (ISBN: 978-3-540-76283-6).

[4] Q. Chen and S. Kawase. On fuzzy-valued fuzzy reasoning. Fuzzy Sets and Systems, 113:237-251, 2000.

[5] S. Coupland and R. John. Geometric type-1 and type-2 fuzzy logic systems. IEEE Transactions on Fuzzy Systems, 15(1):3-15, 2007.

[6] J. C. Figueroa Garcia. An approximation method for type reduction of an interval type- 2 fuzzy set based on $\alpha$-cuts. In Computer Science and Information Systems (FedCSIS), 2012 Federated Conference on, pages 49-54. IEEE, 2012.

[7] J. C. Figueroa Garcia and G. Hernandez. Solving linear programming problems with interval type- 2 fuzzy constraints using interval optimization. In IFSA World Congress and NAFIPS Annual Meeting (IFSA/NAFIPS), 2013 Joint, pages 623-628. IEEE, 2013.
[8] S. Greenfield, F. Chiclana, S. Coupland, and R. John. The collapsing method of defuzzification for discretised interval type-2 fuzzy sets. Information Sciences, 179(13):2055-2069, 2009.

[9] H. Hamrawi and S. Coupland. Non-specificity measures for type2 fuzzy sets. In Proc. FUZZ-IEEE, pages 732-737, Korea, August 2009.

[10] H. Hamrawi and S. Coupland. Type-2 fuzzy arithmetic using alphaplanes. In Proc. IFSA/EUSFLAT, pages 606-611, Portugal, 2009.

[11] H. Hamrawi, S. Coupland, and R. John. Extending operations on type-2 fuzzy sets. In Proc. UKCI, Nottingham, UK, September 2009.

[12] H. Hamrawi, S. Coupland, and R. John. A novel alpha-cut representation for type-2 fuzzy sets. In Proc. FUZZ-IEEE, pages 1-8, Barcelona, Spain, July 2010.

[13] R. John. Type-2 fuzzy sets: an appraisal of theory and applications. International Journal of Uncertainty, Fuzziness and Knowledge-Based Systems, 6:563-576, 1998

[14] R. John and S. Coupland. Type-2 fuzzy logic: A historical view. IEEE Computational Intelligence Magazine, 2(1):57-62, February 2007.

[15] A. Kaufmann and M. Gupta. Introduction to Fuzzy Arithmetic Theory and Applications. Van Nostran Reinhold Co. Inc., 1985.

[16] G. Klir and B. Yuan. Fuzzy Sets and Fuzzy Logic: Theory and Applications. Prentice Hall, Upper Saddle River, NJ, 1995.

[17] F. Liu. An efficient centroid type-reduction strategy for general type-2 fuzzy logic system. Information Sciences, 178(9):2224-2236, 2008.

[18] J. Mendel. Uncertain Rule-Based Fuzzy Logic Systems: Introduction and New Directions. Prentice Hall, Upper Saddle River, NJ, 2001.

[19] J. Mendel, F. Liu, and D. Zhai. $\alpha$-plane representation for type-2 fuzzy sets: Theory and applications. IEEE Transactions on Fuzzy Systems, 17(5):1189-1207, 2009.

[20] J. Mendel and H. Wu. Type-2 fuzzistics for nonsymmetric interval type-2 fuzzy sets: Forward problems. IEEE Transactions on Fuzzy Systems, 15(5):916-930, 2007.

[21] J. M. Mendel. Advances in type-2 fuzzy sets and systems. Information Sciences, 177(1):84-110, January 2007.

[22] J. M. Mendel and R. John. Type-2 fuzzy sets made simple. IEEE Transaction on Fuzzy Systems, 10(2):117-127, 2002.

[23] H. Nguyen. A note on the extension principle for fuzzy sets. $J$. Mathematical Analysis and Applications, 64(2):369-380, 1978.

[24] J. T. Rickard, J. Aisbett, and G. Gibbon. Fuzzy subsethood for fuzzy sets of type-2 and generalized type-n. IEEE Transactions on Fuzzy Systems, 17(1):50-60, 2009.

[25] J. Starczewski. Efficient triangular type-2 fuzzy logic systems. International Journal of Approximate Reasoning, 50(5):799-811, 2009.

[26] H. Tahayori, A. Tettamanzi, and G. Antoni. Approximated type-2 fuzzy set operations. In Proc. FUZZ-IEEE 2006, pages 9042 - 9049, Vancouver, Canada, July 2006.

[27] C. Wagner and H. Hagras. zSlices-Towards bridging the gap between Interval and General Type-2 Fuzzy Logic. In FUZZ-IEEE 2008., pages 489-497, Hong Kong, 2008.

[28] C. Wagner and H. Hagras. Toward general type-2 fuzzy logic systems based on zslices. IEEE Transactions on Fuzzy Systems, 18(4):637 $-660,2010$.

[29] C. Walker and E. Walker. Sets with type-2 operations. International Journal of Approximate Reasoning, 50(1):63-71, 2009.

[30] D. Wu and J. Mendel. Corrections to Aggregation Using the Linguistic Weighted Average and Interval Type-2 Fuzzy Sets. IEEE Transactions on Fuzzy Systems, 16(6):1664-1666, 2008.

[31] D. Wu and J. M. Mendel. Aggregation Using the Linguistic Weighted Average and Interval Type-2 Fuzzy Sets. IEEE Transactions on Fuzzy Systems, 15(6):1145, 2007.

[32] R. R. Yager. Level sets and the extension principle for interval valued fuzzy sets and its application to uncertainy measures. Information Sciences, 178:3565-3576, 2008

[33] L. A. Zadeh. The concept of a linguistic variable and its application to approximate reasoning-1. Information Sciences, 8:199-249, 1975.

[34] L. A. Zadeh. The concept of a linguistic variable and its application to approximate reasoning-2. Information Sciences, 8:301 - 357, 1975.

[35] L. A. Zadeh. The concept of a linguistic variable and its application to approximate reasoning-3. Information Sciences, 9:43 - 80, 1975.

[36] W. Zeng and H. Li. Representation theorem of interval-valued fuzzy set. International Journal of Uncertainty, Fuzziness and KnowledgeBased Systems, 14(3):259-269, 2006.

[37] W. Zeng and Y. Shi. Note on interval-valued fuzzy set. Lecture Notes in Computer Science, 3613:20, 2005. 
TABLE XV

T2FS $\tilde{3}$, In EXAMPLE 5.1. The NUMBERS IN BeTweEn ARE THE SGs, $\tilde{3}_{x}\left(u_{x}\right)$.

\begin{tabular}{|l|c|c|c|c|c|c|c|c|c|c|c|}
\hline$x / u_{x}$ & $\mathbf{0 . 0}$ & $\mathbf{0 . 1}$ & $\mathbf{0 . 2}$ & $\mathbf{0 . 3}$ & $\mathbf{0 . 4}$ & $\mathbf{0 . 5}$ & $\mathbf{0 . 6}$ & $\mathbf{0 . 7}$ & $\mathbf{0 . 8}$ & $\mathbf{0 . 9}$ & $\mathbf{1 . 0}$ \\
\hline 1 & 1.0 & 0.6 & 0.3 & & & & & & & & \\
\hline 2 & & & & 0.1 & 0.6 & 1.0 & 0.7 & 0.2 & & & \\
\hline $\mathbf{3}$ & & & & & & & & & & & 1.0 \\
\hline $\mathbf{4}$ & & & & 0.1 & 0.6 & 1.0 & 0.7 & 0.2 & & & \\
\hline $\mathbf{5}$ & 1.0 & 0.6 & 0.3 & & & & & & & & \\
\hline
\end{tabular}

TABLE XVI

T2FS $\tilde{6}$, In EXAMPLE 5.1. The NUMBERS IN BETWEen ARE THE SGs, $\tilde{6}_{x}\left(u_{x}\right)$.

\begin{tabular}{|l|c|c|c|c|c|c|c|c|c|c|c|}
\hline$x / u_{x}$ & $\mathbf{0 . 0}$ & $\mathbf{0 . 1}$ & $\mathbf{0 . 2}$ & $\mathbf{0 . 3}$ & $\mathbf{0 . 4}$ & $\mathbf{0 . 5}$ & $\mathbf{0 . 6}$ & $\mathbf{0 . 7}$ & $\mathbf{0 . 8}$ & $\mathbf{0 . 9}$ & $\mathbf{1 . 0}$ \\
\hline $\mathbf{4}$ & 1.0 & 0.8 & 0.4 & 0.2 & 0.1 & & & & & & \\
\hline $\mathbf{5}$ & & & & & & 0.2 & 1.0 & 0.4 & & & \\
\hline $\boldsymbol{6}$ & & & & & & & & & & & 1.0 \\
\hline $\mathbf{7}$ & & & & & & 0.2 & 1.0 & 0.4 & & & \\
\hline $\boldsymbol{8}$ & 1.0 & 0.8 & 0.4 & 0.2 & 0.1 & & & & & & \\
\hline
\end{tabular}

TABLE XVII

$\alpha$-PLANE, $\hat{3}_{0.2}$, IN EXAMPLE 5.1 .

\begin{tabular}{|l|ccccc|}
\hline$x$ & 1 & 2 & 3 & 4 & 5 \\
\hline$\tilde{3}_{0.2}(x)$ & {$[0,0.2]$} & {$[0.4,0.7]$} & {$[1,1]$} & {$[0.4,0.7]$} & {$[0,0.2]$} \\
\hline
\end{tabular}

TABLE XVIII

$\alpha$-PLANE, $\tilde{6}_{0.2}$, IN EXAMPLE 5.1 .

\begin{tabular}{|l|ccccc|}
\hline$x$ & 4 & 5 & 6 & 7 & 8 \\
\hline$\tilde{6}_{0.2}(x)$ & {$[0,0.3]$} & {$[0.5,0.7]$} & {$[1,1]$} & {$[0.5,0.7]$} & {$[0,0.3]$} \\
\hline
\end{tabular}

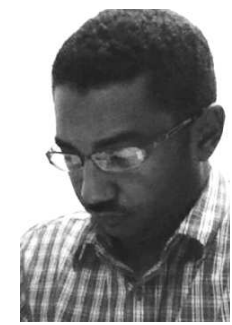

Hussam Hamrawi Hussam Hamrawi has been Assistant Professor of Computer and Systems Engineering at AAUT, Merowe, Sudan since May 2015. Prior to this he held the post of Assistant Professor of Intelligent Systems at the University of Bahri, Sudan from September 2011. His area of expertise is fuzzy logic, obtaining his $\mathrm{PhD}$ in this subject from De Montfort University in 2011.

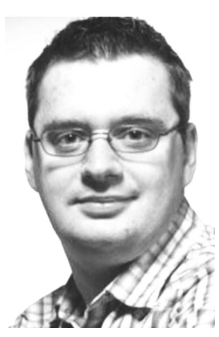

Simon Coupland Simon Coupland has worked at the Centre for Computational Intelligence at De Montfort University, UK since 2005, completing his $\mathrm{PhD}$ there in 2006. His area of expertise is type-2 fuzzy logic and application of computational intelligence approach more generally. He has chaired many conference session on type- 2 fuzzy logic and reviews paper for a number of international journals in this field.

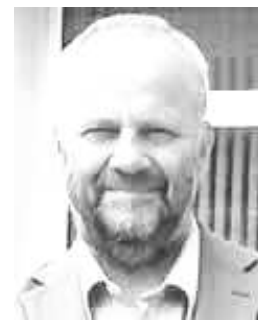

Robert John Professor Robert John joined the School of Computer Science at University of Nottingham in 2013 as the Head of the Automated Scheduling, Optimisation and Planning Group (ASAP) and a member of the Lab for Uncertainty in Data and Decision Making (LUCID). Prior to joining ASAP he worked for 24 years at De Montfort University as a Professor, leading a research group in Computational Intelligence as well as holding various senior management roles. Before being an academic he had a career in industry working for British Gas and software companies in Artificial Intelligence. Robert has an international reputation for his work on fuzzy logic having over 150 publications with over 5000 citations. He has organised and chaired international conferences on Computational Intelligence and is a senior member of IEEE. 
TABLE XIX

The $\alpha$-Cuts of $\alpha$-Planes, $\tilde{3}_{0.2}$ AND $\tilde{6}_{0.2}$, IN EXAMPle 5.1.

\begin{tabular}{|l|c|c|c|c|}
\hline$\alpha$ & $\underline{3}_{0.2, \alpha}$ & $\underline{6}_{\tilde{0.2, \alpha}}$ & $\overline{3}_{0.2, \alpha}$ & $\overline{6}_{0.2, \alpha}$ \\
\hline 0.0 & $\{1,2,3,4,5\}$ & $\{4,5,6,7,8\}$ & $\{1,2,3,4,5\}$ & $\{4,5,6,7,8\}$ \\
0.1 & $\{2,3,4\}$ & $\{5,6,7\}$ & $\{1,2,3,4,5\}$ & $\{4,5,6,7,8\}$ \\
0.2 & $\{2,3,4\}$ & $\{5,6,7\}$ & $\{1,2,3,4,5\}$ & $\{4,5,6,7,8\}$ \\
0.3 & $\{2,3,4\}$ & $\{5,6,7\}$ & $\{2,3,4\}$ & $\{4,5,6,7,8\}$ \\
0.4 & $\{2,3,4\}$ & $\{5,6,7\}$ & $\{2,3,4\}$ & $\{5,6,7\}$ \\
0.5 & $\{3\}$ & $\{5,6,7\}$ & $\{2,3,4\}$ & $\{5,6,7\}$ \\
0.6 & $\{3\}$ & $\{6\}$ & $\{2,3,4\}$ & $\{5,6,7\}$ \\
0.7 & $\{3\}$ & $\{6\}$ & $\{2,3,4\}$ & $\{5,6,7\}$ \\
0.8 & $\{3\}$ & $\{6\}$ & $\{3\}$ & $\{6\}$ \\
0.9 & $\{3\}$ & $\{6\}$ & $\{3\}$ & $\{6\}$ \\
1.0 & $\{3\}$ & $\{6\}$ & $\{3\}$ & $\{6\}$ \\
\hline
\end{tabular}

TABLE XX

THE $\alpha$-CUTS OF $\alpha$-PLANES, $\tilde{3}_{0 \sim 2} \cup \tilde{6}_{0.2}$, IN EXAMPLe 5.1.

\begin{tabular}{|l|c|c|}
\hline$\alpha$ & $\underline{3}_{0.2, \alpha} \cup \underline{6}_{0.2, \alpha}$ & $\overline{3}_{0.2, \alpha} \cup \overline{6}_{0.2, \alpha}$ \\
\hline 0.0 & $\{1,2,3,4,5,6,7,8\}$ & $\{1,2,3,4,5,6,7,8\}$ \\
0.1 & $\{2,3,4,5,6,7\}$ & $\{1,2,3,4,5,6,7,8\}$ \\
0.2 & $\{2,3,4,5,6,7\}$ & $\{1,2,3,4,5,6,7,8\}$ \\
0.3 & $\{2,3,4,5,6,7\}$ & $\{2,3,4,5,6,7,8\}$ \\
0.4 & $\{2,3,4,5,6,7\}$ & $\{2,3,4,5,6,7\}$ \\
0.5 & $\{3,5,6,7\}$ & $\{2,3,4,5,6,7\}$ \\
0.6 & $\{3,6\}$ & $\{2,3,4,5,6,7\}$ \\
0.7 & $\{3,6\}$ & $\{2,3,4,5,6,7\}$ \\
0.8 & $\{3,6\}$ & $\{3,6\}$ \\
0.9 & $\{3,6\}$ & $\{3,6\}$ \\
1.0 & $\{3,6\}$ & $\{3,6\}$ \\
\hline
\end{tabular}

TABLE XXI

$\alpha$-PLANE, $\tilde{3}_{0.2, \alpha} \cup \tilde{6}_{0.2, \alpha}$, IN EXAMPLE 5.1 FROM ITS $\alpha$-CUTS IN TABLE XX.

\begin{tabular}{|c|l|l|l|}
\hline$x$ & $\alpha\left(\underline{3}_{\tilde{0} 2, \alpha} \cup \underline{6}_{0.2, \alpha}\right)(x)$ & $\alpha\left(\underline{3}_{0.2, \alpha} \cup \underline{6}_{0 \tilde{2}, \alpha}\right)(x)$ & $\left(\tilde{3}_{0 \tilde{2}, \alpha} \cup \tilde{\sigma_{0.2, \alpha}}\right)(x)$ \\
\hline 1 & 0 & $0,0.1,0.2$ & {$[0.2]$} \\
2 & $0,0.1,0.2,0.3,0.4$ & $0,0.1,0.2,0.3,0.4,0.5,0.6,0.7$ & {$[0.4,0.7]$} \\
3 & $0,0.1,0.2,0.3,0.4,0.5,0.6,0.7,0.8,0.9,1$ & $0,0.1,0.2,0.3,0.4,0.5,0.6,0.7,0.8,0.9,1$ & {$[1,1]$} \\
4 & $0,0.1,0.2,0.3,0.4$ & $0,0.1,0.2,0.3,0.4,0.5,0.6,0.7$ & {$[0.4,0.7]$} \\
5 & $0,0.1,0.2,0.3,0.4,0.5$ & $0,0.1,0.2,0.3,0.4,0.5,0.6,0.7$ & {$[0.5,0.7]$} \\
6 & $0,0.1,0.2,0.3,0.4,0.5,0.6,0.7,0.8,0.9,1$ & $0,0.1,0.2,0.3,0.4,0.5,0.6,0.7,0.8,0.9,1$ & {$[1,1]$} \\
7 & $0,0.1,0.2,0.3,0.4,0.5$ & $0,0.1,0.2,0.3,0.4,0.5,0.6,0.7$ & {$[0.5,0.7]$} \\
8 & 0 & $0,0.1,0.2,0.3$ & {$[0,0.3]$} \\
\hline
\end{tabular}

